\title{
Bose-Einstein Condensate Dark Matter Halos Confronted with Galactic Rotation Curves
}

\author{
M. Dwornik, Z. Keresztes, E. Kun, and L. Á. Gergely \\ Institute of Physics, University of Szeged, Dóm Tér 9, Szeged 6720, Hungary \\ Correspondence should be addressed to L. Á. Gergely; laszlo.a.gergely@gmail.com
}

Received 7 October 2016; Revised 15 December 2016; Accepted 25 December 2016; Published 8 February 2017

Academic Editor: Sergei D. Odintsov

Copyright (C) 2017 M. Dwornik et al. This is an open access article distributed under the Creative Commons Attribution License, which permits unrestricted use, distribution, and reproduction in any medium, provided the original work is properly cited. The publication of this article was funded by SCOAP S $^{3}$

We present a comparative confrontation of both the Bose-Einstein Condensate (BEC) and the Navarro-Frenk-White (NFW) dark halo models with galactic rotation curves. We employ 6 High Surface Brightness (HSB), 6 Low Surface Brightness (LSB), and 7 dwarf galaxies with rotation curves falling into two classes. In the first class rotational velocities increase with radius over the observed range. The BEC and NFW models give comparable fits for HSB and LSB galaxies of this type, while for dwarf galaxies the fit is significantly better with the BEC model. In the second class the rotational velocity of HSB and LSB galaxies exhibits long flat plateaus, resulting in better fit with the NFW model for HSB galaxies and comparable fits for LSB galaxies. We conclude that due to its central density cusp avoidance the BEC model fits better dwarf galaxy dark matter distribution. Nevertheless it suffers from sharp cutoff in larger galaxies, where the NFW model performs better. The investigated galaxy sample obeys the Tully-Fisher relation, including the particular characteristics exhibited by dwarf galaxies. In both models the fitting enforces a relation between dark matter parameters: the characteristic density and the corresponding characteristic distance scale with an inverse power.

\section{Introduction}

The visible part of most galaxies is embedded in a dark matter (DM) halo of yet unknown composition, observable only through its gravitational interaction with the baryonic matter. Assuming the standard $\Lambda \mathrm{CDM}$ cosmological model, the Planck satellite measurements of the cosmic microwave background anisotropy power spectrum support $4.9 \%$ baryonic matter, $26.8 \% \mathrm{DM}$, and $68.3 \%$ dark energy in the Universe $[1,2]$.

Investigation of mass distribution of spiral galaxies is an essential tool in the research of DM. Beside the stellar disk and central bulge, most of the galaxies harbour a spherically symmetric, massive DM halo, which dominates the dynamics in the outer regions of the stellar disk. Nevertheless there are examples of galaxies which at larger radii are better described by a flattened baryonic mass distribution (global disk model) [3].

Several DM candidates and alternatives have been proposed, the latter assuming Einstein's theory of gravity breaking down on the galactic scale and above ([4-12]). In brane-world and $f(R)$-gravity models, the galactic rotation curves could be explained without DM ([13-16]).

At this moment there are strong experimental constraints for all proposed dark matter candidates. Supersymmetric dark matter has been strongly constrained by LHC [17, 18], sterile neutrinos disruled with $99 \%$ confidence level by IceCube [19], Weekly Interacting Massive Particles (WIMPs) severely bounded by the LUX [20], PandaX-II [21], and Xenon100 [22] experiments. Extra dimensional effects as dark matter substitutes have been also contained by LHC [23]. Massive Compact Halo Objects (MACHOs) with masses below 20 solar masses have been shown to give at most $10 \%$ of dark matter by microlensing experiments on the Large Magellanic Cloud [24]. There is still hope for larger mass MACHOs as dark matter candidates, revived after the spectacular first direct detection of gravitational waves [25], sourced by black holes of approximately 30 solar masses.

It is well known that hot dark matter (HDM) consisting of light $(m \propto \mathrm{eV})$ particles cannot reproduce the cosmological structure formation, as they imply that the superclusters of galaxies are the first structures to form contradicting $\mathrm{CMB}$ 
observations, according to which superclusters would form at the present epoch [26]. Warm dark matter $(m \propto \mathrm{keV})$ models seem to be compatible with the astronomical observations on galactic and also cosmological scales [27, 28]. Leading candidates for warm dark matter are the right handed neutrinos, which in contrast with their left handed counterparts do not participate in the weak interaction. The decay of these sterile neutrinos produces high amount of X-rays, which can boost the star formation rate leading to an earlier reionization [29]. The existence of sterile neutrinos was however severely constrained by recent IceCube Neutrino Observatory experiments [30]. Cold dark matter (CDM) also shows remarkably good agreement with observations over kpc scales $([31,32])$. Particular CDM candidates, like neutralinos (which are stable and can be produced thermally in the early Universe) and other WIMPs originating in supersymmetric extensions of the Standard Model were severely constrained by recent LHC results, rendering them into the range $200 \mathrm{GeV} \lesssim m_{n} \lesssim$ $500 \mathrm{GeV}$ [33]. In a Higgs-portal DM scenario the Higgs boson acts as the mediator particle between DM and Standard Model particles, and it can decay to a pair of DM particles. Very recent constraints established by the ATLAS Collaboration on DM-nucleon scattering cross-section impose upper limits of approximately $60 \mathrm{GeV}$ for each of the scalar, fermion, and vector DM candidates (see Figure 4 of [34]), within the framework of this scenario. While MACHOs of masses less than 10 solar masses (like white dwarfs, neutron stars, brown dwarfs and unassociated planets, and primordial black holes in the astrophysical mass range) were disruled either by Big Bang Nucleosynthesis constraints or microlensing experiments as dominant DM candidates, primordial black holes with intermediate mass could still be viable candidates $[35,36]$.

Large $N$-body simulations (e.g., [39]) performed in the framework of the $\Lambda \mathrm{CDM}$-model ( $\Lambda$ being the cosmological constant) were compatible with CDM halos with central density cusps [40]. They are modeled by the Navarro-FrenkWhite (NFW) DM density profile $\rho_{\mathrm{NFW}}(r)=\rho_{s} /\left(r / r_{s}\right)(1+$ $\left.r / r_{s}\right)^{2}$, where $r_{s}$ is a scale radius and $\rho_{s}$ is a characteristic density. Some observations support such a steep cuspy density profile $[41,42]$; nevertheless certain high-resolution rotation curves instead indicate that the distribution of DM in the centres of DM dominated dwarf and Low Surface Brightness (LSB) galaxies is much shallower, exhibiting a core with nearly constant density [43]. In turn, the baryonic matter distribution may also affect the DM density profile. As shown in [44] a dark matter core within an isolated, initially cuspy dark matter halo may form due to strong supernova feedback. By contrast, adiabatic contraction of baryonic gas tends to produce even cuspier dark matter halos [45].

The surface number-density profiles of satellites decline with the projected distance as a power law with the slope $(-2) \div(-1.5)$, while the line-of-sight velocity dispersion declines gradually [46]. These observations support the NFW model on scales of $50 \div 500 \mathrm{kpc}$.

In a cosmological setup various scalar field DM models were also discussed ([47,48] and references therein). A particular scalar field DM model describes light bosons in a dilute gas. The thermal de Broglie wavelength of the particles is $\lambda_{T} \propto 1 / \sqrt{m T}$, which can be large for light bosons $(m<$ $\mathrm{eV}$ ) and for low temperature. Below a critical temperature $\left(T_{c}\right)$, the bosons' wave packets, which are the order of $\lambda_{T}$ overlap, result in correlated particles. Such bosons share the same quantum ground state, behaving as a Bose-Einstein Condensate (BEC), characterized by a single macroscopic wave function. It has been proposed that galactic DM halos could be gigantic BECs [49].

It has been shown that caustics of ring shape appear in rotating BEC models, which have an effect on rotation curves, by causing bumps [50, 51]. Such ring shaped caustics degenerate into the origin in the nonrotating BEC limit, adopted in this paper.

The self-gravitating condensate is described by the GrossPitaevskii-Poisson equation system in the mean-field approximation [10, 52-54]. In the Thomas-Fermi approximation, a 2-parameter (mass $m$ and scattering length $a$ ) density distribution of the BEC halo is obtained [see (3) below], which is less concentrated towards the centre as compared to the NFW model, relaxing the cuspy halo problem.

In model [55] where a normal dark matter phase with an equation of state $P=\rho c^{2} \sigma_{\mathrm{tr}}^{2}$ condensed into a BEC with selfinteraction $\left(\sigma_{\mathrm{tr}}=0.0017\right.$ being the one-dimensional velocity dispersion and $c$ the speed of light), the stability of the BEC halo depends on the particle mass and scattering length. For a given mass the stability occurs for larger scattering length and for given scattering length the stability appears at smaller mass. For the following scattering lengths: $a=10^{3} \mathrm{fm}, a=$ $10^{-14} \mathrm{fm}$, and $a=10^{-55} \mathrm{fm}$, the mass of the BEC particle arises as $m>1 \mathrm{eV}, m>2 \times 10^{-6} \mathrm{eV}$, and $m>4.57 \times 10^{-20} \mathrm{eV}$, respectively. Galactic size stable halos can form with $m>$ $10^{-24} \mathrm{eV}$ (Figure 3 in [56]).

A stable BEC halo can form as a result of gravitational collapse [57]. The model has been tested on kpc scales confronting it with galactic rotation curve observations [10]. It was pointed out by [58] that the effects of BEC DM should be seen in the matter power spectrum if the boson mass is in the range $15 \mathrm{meV}<m<35 \mathrm{meV}$ and $300 \mathrm{meV}<m<$ $700 \mathrm{meV}$ for the scattering lengths $a=10^{6} \mathrm{fm}$ and $a=$ $10^{10} \mathrm{fm}$, respectively. In [59] the authors showed that the observed collisional behaviour of DM in Abell 520 cluster can also be recovered within the framework of the BEC model. All of the mentioned BEC particle masses are consistent with the limit $m<1.87 \mathrm{eV}$ imposed from galaxy observations and $N$-body simulation [60]. A discrepancy was however pointed out between the best fit density profile parameters derived from the strong lensing and the galactic rotational curves data. In conclusion the BEC halo should be denser in lens galaxies than in dwarf spheroidals [61].

In this work we critically examine the BEC model as a possible DM candidate against rotation curve data, pointing out both advantages and disadvantages over the NFW model. Previous studies on the compatibility of the BEC model and galactic rotation curves were promising but relied on a less numerous and less diversified set of galaxies than employed here $([62,63])$. The paper has the following structure. The basic properties of the BEC DM model are reviewed in 
Section 2. In Section 3 a comparison is made between the theoretical predictions of the BEC model and the observed rotation curve data of three types of galaxies: the High Surface Brightness (HSB), LSB, and dwarf galaxies. The conclusions are presented in Section 4.

\section{The Bose-Einstein Condensate Galactic Dark Matter Halo}

An ideal, dilute Bose gas at very low temperature forms a Bose-Einstein Condensate in which all particles are in the same ground state. In the thermodynamic limit, the critical temperature for the condensation is $T_{c}=2 \pi \hbar^{2}(n / \zeta)^{3 / 2} / m k_{B}$ [64]. Here $n$ and $m$ are the number density and the mass of the bosons, respectively, and $\zeta=2.612$ is a constant, while $\hbar$ and $k_{B}$ denote the reduced Planck and Boltzmann constants, respectively. Atoms can be regarded as quantummechanical wave packets of the order of their thermal de Broglie wavelength $\lambda_{T}=\sqrt{2 \pi \hbar^{2} /\left(m k_{B} T\right)}$. The condition for the condensation $T<T_{c}$ can be reformulated as $l<\lambda_{T} / \zeta^{-1 / 3}$, where $l$ is the average distance between pairs of bosons, and it occurs when the temperature, hence the momentum of the bosons, decreases and as a consequence their de Broglie wavelengths overlap. The thermodynamic limit is only approximately realized, the finite size giving corrections to the critical temperature [65-68]. A dilute, nonideal Bose gas also displays BEC; on the other hand, the condensate fraction is smaller than unity at zero temperature and the critical temperature is also modified [69-72]. Experimentally, BEC (which could be formed by bosonic atoms but also form fermionic Cooper pairs) has been realized first in ${ }^{87} \mathrm{Rb}[73-$ 75], then in ${ }^{23} \mathrm{Na}[76,77]$, and in ${ }^{7} \mathrm{Li}[78]$.

In a dilute gas, only two-particle interactions dominate. The repulsive, two-body interparticle potential is approximated as $V_{\text {self }}=\lambda \delta\left(\mathbf{r}-\mathbf{r}^{\prime}\right)$, with a self-coupling constant $\lambda=4 \pi \hbar^{2} a / m$, where $a$ is the scattering length. Then in the mean-field approximation (in case when we neglect the contribution of the excited states) the BEC is described by the Gross-Pitaevskii equation [52-54]:

$$
\begin{aligned}
i \hbar & \frac{\partial}{\partial t} \psi(\mathbf{r}, t) \\
\quad & {\left[-\frac{\hbar^{2}}{2 m} \Delta+V_{\text {self grav }}(\mathbf{r})+\lambda \rho(\mathbf{r}, t)\right] \psi(\mathbf{r}, t), }
\end{aligned}
$$

where $\psi(\mathbf{r}, t)$ is the wave function of the condensate and $\Delta$ is the 3-dimensional Laplacian. The probability density $\rho(\mathbf{r}, t)=$ $|\psi(\mathbf{r}, t)|^{2}$ is normalized to

$$
n_{0}(t)=\int d \mathbf{r} \rho(\mathbf{r}, t),
$$

where $n_{0}(t)$ is the number of particles in the ground state and $\rho(\mathbf{r}, t)$ is the number density of the condensate. The potential $V_{\text {self grav }}(\mathbf{r}) / m$ is the Newtonian gravitational potential produced by the Bose-Einstein Condensate.

Stationary solutions of the Gross-Pitaevskii equation can be found in a simple way by using the Madelung representation of complex wave functions $[79,80]$, then deriving the Madelung hydrodynamic equations [79]. Madelung's equations can be interpreted as the continuity and Euler equations of fluid mechanics, with quantum corrections included. However, the quantum correction potential in the generalized Euler equation contributes significantly only close to the boundary of the system [81]. In the Thomas-Fermi approximation the quantum correction potential is neglected compared to the self-interaction term. This approximation becomes more accurate as the particle number increases [82].

Assuming a spherically symmetric distribution of the condensate the following solution was found $[10,81]$ :

$$
\rho_{\mathrm{BEC}}(r)=\rho_{\mathrm{BEC}}^{(c)} \frac{\sin k r}{k r},
$$

where $\rho_{\mathrm{BEC}}=m \rho(r)$ and

$$
k=\sqrt{\frac{G m^{3}}{\hbar^{2} a}} .
$$

The central density $\rho_{\mathrm{BEC}}^{(c)} \equiv \rho_{\mathrm{BEC}}(0)$ is determined from normalization condition (2) as

$$
\rho_{\mathrm{BEC}}^{(c)}=\frac{n_{0} m k^{3}}{4 \pi^{2}} .
$$

The Thomas-Fermi approximation remains valid for $n_{0} \gg$ $1 / k a[81]$.

The BEC galactic DM halo's size is defined by $\rho\left(R_{\mathrm{BEC}}\right)=0$, giving $k=\pi / R_{\mathrm{BEC}}$, that is,

$$
R_{\mathrm{BEC}}=\pi \sqrt{\frac{\hbar^{2} a}{G m^{3}}} .
$$

The mass profile of the BEC halo is then given as

$$
\begin{aligned}
m_{\mathrm{BEC}}(r) & =4 \pi \int_{0}^{r} \rho_{\mathrm{BEC}}(r) r^{2} d r \\
& =\frac{4 \pi \rho_{\mathrm{BEC}}^{(c)}}{k^{2}} r\left(\frac{\sin k r}{k r}-\cos k r\right) .
\end{aligned}
$$

The BEC halo contributes to the velocity profile of the particles which are moving on circular orbit as dictated by the Newtonian gravitational force [10]. This can be taken into account by the following equation:

$$
v^{2}(r)=\frac{4 \pi G \rho_{\mathrm{BEC}}^{(c)}}{k^{2}}\left(\frac{\sin k r}{k r}-\cos k r\right),
$$

which needs to be added to the baryonic contribution, respectively.

\section{Confronting the Model with Rotation Curve Data}

The validity of our model was tested by confronting the rotation curve data of a sample of $6 \mathrm{HSB}, 6 \mathrm{LSB}$, and 7 dwarf galaxies, with both the NFW DM and the BEC density 
profiles. For reasons to become obvious during our analysis, we split both the HSB and LSB data sets into two groups (type I. and II.), based on the shapes of the curves. In the first group the rotational velocities increase over the whole observed range, while in the second set the rotation curves exhibit long flat regions.

The commonly used NFW model has the mass density profile

$$
\rho_{\mathrm{NFW}}(r)=\frac{\rho_{s}}{\left(r / r_{s}\right)\left(1+r / r_{s}\right)^{2}},
$$

where $\rho_{s}$ and $r_{s}$ are a characteristic density and distance scale, to be determined from the fit.

The mass within a sphere with radius $r=y r_{s}$ is then given as

$$
m_{\mathrm{NFW}}(r)=4 \pi \rho_{s} r_{s}^{3}\left[\ln (1+y)-\frac{y}{1+y}\right],
$$

where $y$ is a positive dimensionless radial coordinate.

3.1. HSB Galaxies. In this subsection we will follow the method described in [15]. In HSB galaxy the baryonic component was decomposed into a thin stellar disk and a spherically symmetric bulge. It was assumed that the mass distribution of bulge component follows the deprojected luminosity distribution with a factor known as the mass-to-light ratio. The bulge parameters were estimated from a Sérsic $r^{1 / n}$ bulge model, which was obtained by the fitting of the optical $I$-band galaxy light profiles.

Each galaxy's spheroidal bulge component has a surface brightness profile which is described by a generalized Sérsic function [83];

$$
I_{b}(r)=I_{0, b} \exp \left[-\left(\frac{r}{r_{0}}\right)^{1 / n}\right],
$$

wherein $I_{0, b}$ is the central surface brightness of the bulge, $r_{0}$ is the characteristic radius of the bulge, and the magnituderadius curve's shape parameter is denoted by $n$.

The mass-to-light ratio for the Sun is $\gamma_{\odot}=5133 \mathrm{~kg} \mathrm{~W}^{-1}$. The mass-to-light ratio of the bulge $\sigma$ will be given in units of $\gamma_{\odot}$ (solar units). We will also give the mass in units of the solar mass $M_{\odot}=1.98892 \times 10^{30} \mathrm{~kg}$. We assume that the radial distribution of visible mass follows the radial distribution of light derived from the bulge-disk decomposition. Accordingly the mass of the bulge inside the projected radius $r$ can be derived from the surface brightness observed within this radius:

$$
m_{b}(r)=\sigma \frac{\mathcal{N}(D)}{F_{\odot}} 2 \pi \int_{0}^{r} I_{b}(r) r d r
$$

where $F_{\odot}(D)$ is the apparent flux density of the Sun at a distance $D \mathrm{Mpc}, F_{\odot}(D)=2.635 \times 10^{6-0.4 f_{\odot}} \mathrm{mJy}$, with $f_{\odot}=$ $4.08+5 \lg (D / 1 \mathrm{Mpc})+25 \mathrm{mag}$, and

$$
\mathcal{N}(D)=4.4684 \times 10^{-35} \mathrm{D}^{-2} \mathrm{~m}^{-2} \operatorname{arcsec}^{2} .
$$

The rotational velocity related to the bulge is

$$
v_{b}^{2}(r)=\frac{G m_{b}(r)}{r}
$$

where $G$ is the gravitational constant.

In case of a spiral galaxy, the radial surface brightness profile of the disk decreases exponentially as a function of the radius [84];

$$
I_{d}(r)=I_{0, d}^{\mathrm{HSB}} \exp \left(-\frac{r}{h^{\mathrm{HSB}}}\right),
$$

where $I_{0, d}^{\mathrm{HSB}}$ is the central surface brightness of the disk and $h^{\mathrm{HSB}}$ is a characteristic disk length scale. The disk contributes to the circular velocity as follows ([84]):

$$
v_{d}^{2}(x)=\frac{G M_{D}^{\mathrm{HSB}}}{2 h^{\mathrm{HSB}}} x^{2}\left(I_{0} K_{0}-I_{1} K_{1}\right),
$$

where $x=r / h^{\mathrm{HSB}}$ and $I_{n}$ and $K_{n}$ are the modified Bessel functions evaluated at $x / 2$, while $M_{D}^{\mathrm{HSB}}$ is the total mass of the disk.

Accordingly in HSB galaxy the rotational velocity adds up as

$$
v_{\mathrm{tg}}^{2}(x)=v_{b}^{2}(x)+v_{d}^{2}(x)+v_{\mathrm{DM}}^{2}(x)
$$

In order to validate the BEC+baryonic model, we confront it with rotation curve data of 6 well-tested galaxies (which were already employed in [15] for testing a braneworld model). The data was obtained from a sample given in [37] and meets the following criteria: (i) it has to be among the best accuracies obtained from the sample and (ii) the bulge has to be spherically symmetric. As a check we also fitted the NFW + baryonic model with the same data set. The respective rotation curves are plotted for both models on Figures 1 and 2. The small humps on both figures are due to the baryonic component. From the available photometric data the best fitting values were derived for the baryonic model parameters $I_{0, b}, n, r_{0}, r_{b}$, and $I_{0, d}^{\mathrm{HSB}} h^{\mathrm{HSB}}$. By fitting BEC and NFW models to the investigated rotation curve data, the parameters for these models (as well as the corresponding baryonic parameters) were calculated. The parameter values are indicated in Tables 1 and 2.

Both the BEC and NFW DM models give comparable $\chi_{\min }^{2}$ values (within $1 \sigma$ confidence level) for HSB I galaxies. In case of galaxies with extended flat regions (HSB II), the NFW DM model fits better the rotation curves; nevertheless BEC model gives rotational curves which fall outside the $1 \sigma$ confidence level.

3.2. LSB Galaxies. The surface brightness of LSB galaxies is substantially fainter than the brightness of the sky at night. They belong to an early stage class of galaxies [85]. LSB galaxies were found to be metal poor, which indicates a lower star formation rate than what is generally found in HSB galaxies [86]. Wide spectrum of colors can be measured in case of LSB galaxies ranging from red to blue [87] and they are diverse as regards morphologies and other properties. 
TABLE 1: The distances $(D)$ and the photometric parameters of the 6 HSB galaxy sample as determined by the fit with available photometric data [37]. Bulge parameters: the central surface brightness $\left(I_{0, b}\right)$, the shape parameter $(n)$, the characteristic radius $\left(r_{0}\right)$, and radius of the bulge $\left(r_{b}\right)$. Disk parameters: central surface brightness $\left(I_{0, d}^{\mathrm{HSB}}\right)$ and length scale $\left(h^{\mathrm{HSB}}\right)$ of the disk.

\begin{tabular}{lcccccc}
\hline Galaxy & $\begin{array}{c}D \\
\mathrm{Mpc}\end{array}$ & $\begin{array}{c}I_{0, b} \\
\mathrm{mJy} / \mathrm{arcsec}^{2}\end{array}$ & $n$ & $\begin{array}{c}r_{0} \\
\mathrm{kpc}\end{array}$ & $\begin{array}{c}r_{b} \\
\mathrm{kpc}\end{array}$ & $\begin{array}{c}I_{0, d}^{\mathrm{HSB}} \\
\mathrm{mJy}^{2} \mathrm{arcsec}^{2}\end{array}$ \\
\hline ESO215G39 & 61.29 & 0.1171 & 0.6609 & 0.78 & 2.58 & 0.0339 \\
ESO322G77 & 38.19 & 0.1949 & 0.7552 & 0.33 & 1.37 & 0.0744 \\
ESO509G80 & 92.86 & 0.2090 & 0.7621 & 1.10 & 4.69 & 0.0176 \\
\hline ESO323G25 & 59.76 & 0.1113 & 0.4626 & 0.43 & 0.99 & 0.0825 \\
ESO383G02 & 85.40 & 0.6479 & 0.7408 & 0.42 & 1.94 & 0.5118 \\
ESO446G01 & 98.34 & 0.2093 & 0.8427 & 1.28 & 6.33 & 3.47 \\
\hline
\end{tabular}
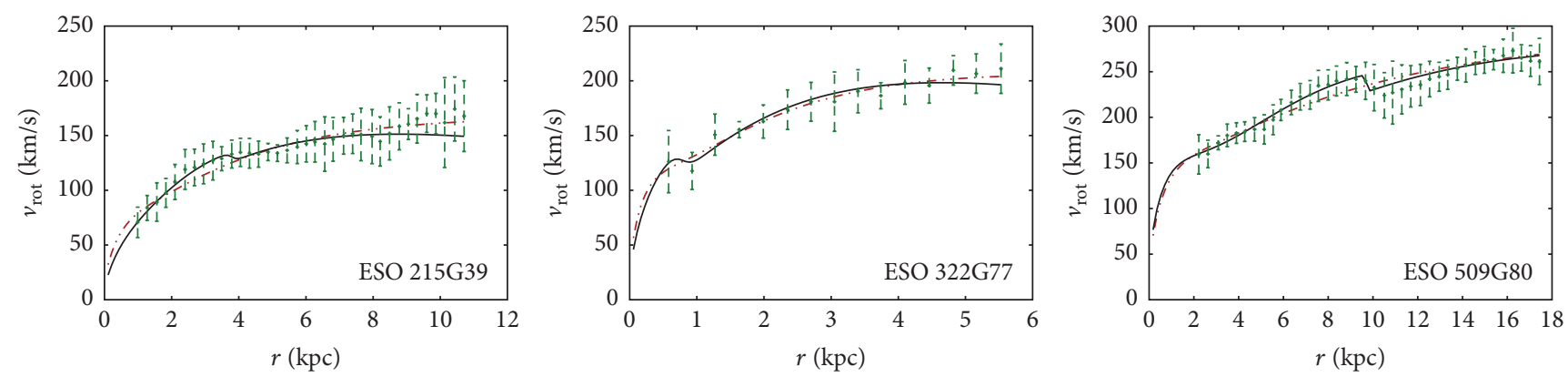

FIGURE 1: Best fit curves for the HSB I. galaxy sample where the solid black lines hold for the baryonic matter + BEC model, while the dashed red lines refer to the baryonic matter + NFW model.
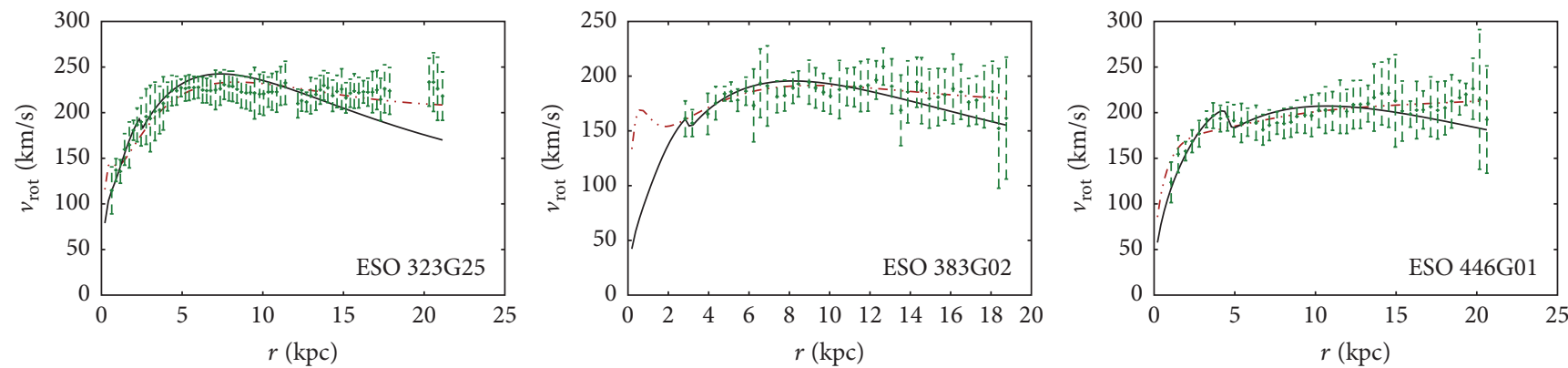

FIgURE 2: Best fit curves for the HSB II. galaxy sample. The solid black lines hold for the baryonic matter + BEC model, while the dashed red lines hold for the baryonic matter + NFW model. The BEC model does not describe well the extended flat regions.

Most of the LSB galaxies that were observed are dwarf galaxies; however there is also a significant number of large spirals among LSB galaxies [88].

According to our model the LSB galaxy is made up of two main components, one being a thin stellar + gas disk and the other one being a CDM component which is assumed to be a $\mathrm{BEC}$. We use the same model for the disk component as in the case of the HSB galaxies. The surface brightness profile can be described by the following equation [84]:

$$
I_{d}(r)=I_{0, d}^{\mathrm{LSB}} \exp \left(-\frac{r}{h^{\mathrm{LSB}}}\right),
$$

where $I_{0, d}^{\mathrm{LSB}}$ is the central surface brightness and $h^{\mathrm{LSB}}$ is the disk length scale. The contribution of the disk to the circular velocity can be expressed as

$$
v_{d}^{2}(r)=\frac{G M_{D}^{\mathrm{LSB}}}{2 h^{\mathrm{LSB}}} q^{2}\left(I_{0} K_{0}-I_{1} K_{1}\right),
$$

where $q=r / h^{\mathrm{LSB}}$ and $M_{D}^{\mathrm{LSB}}$ are the total mass of the disk while the modified Bessel functions $I_{n}$ and $K_{n}$ are evaluated at $q / 2$.

Consequently, for an arbitrary projected radius $r$ the rotational velocity can be calculated based on the combined model resulting in the following equation:

$$
v_{\mathrm{tg}}^{2}(r)=v_{d}^{2}(r)+v_{\mathrm{DM}}^{2}
$$

A preliminary check confirmed that the $\mathrm{BEC}+$ baryonic model represents a better fit than the purely BEC model.

We confronted the BEC model with 6 LSB galaxies chosen from a larger sample [38]. The applied data were obtained from both $H I$ and $H \alpha$ measurements. From a $\chi^{2}$-test the parameters in both the BEC + baryonic and NFW + baryonic models were identified: these are shown in Table 3 . The best fit rotation curves are represented on Figures 3 and 4 . 


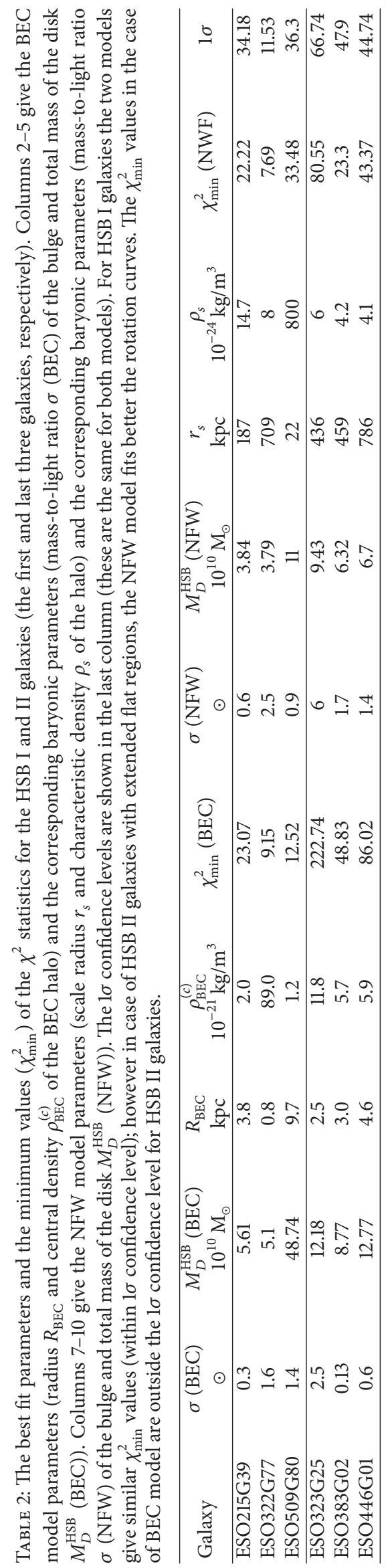




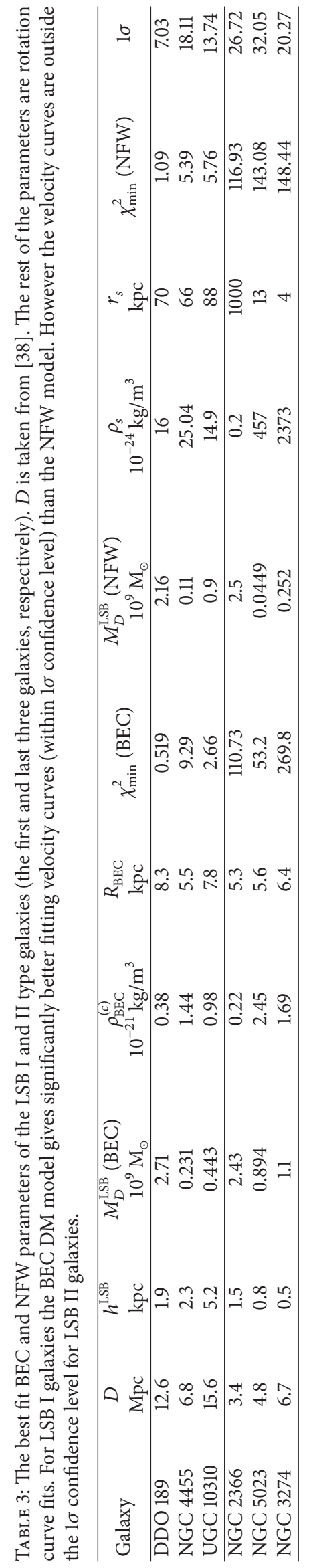



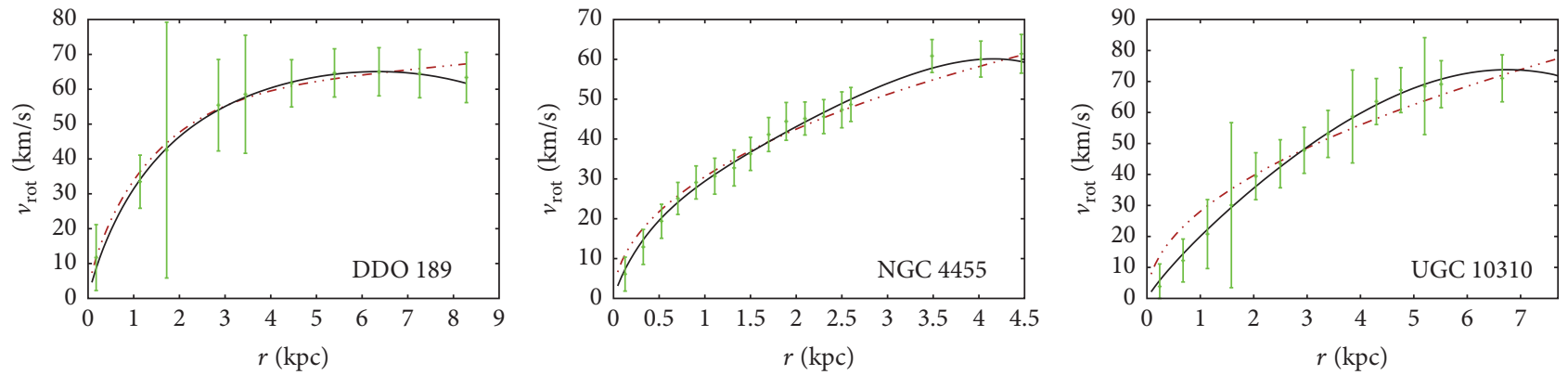

Figure 3: Best fit curves for the LSB I. galaxy sample. The solid black lines indicate the baryonic matter + BEC model, while the dashed red lines indicate the baryonic matter + NFW model.
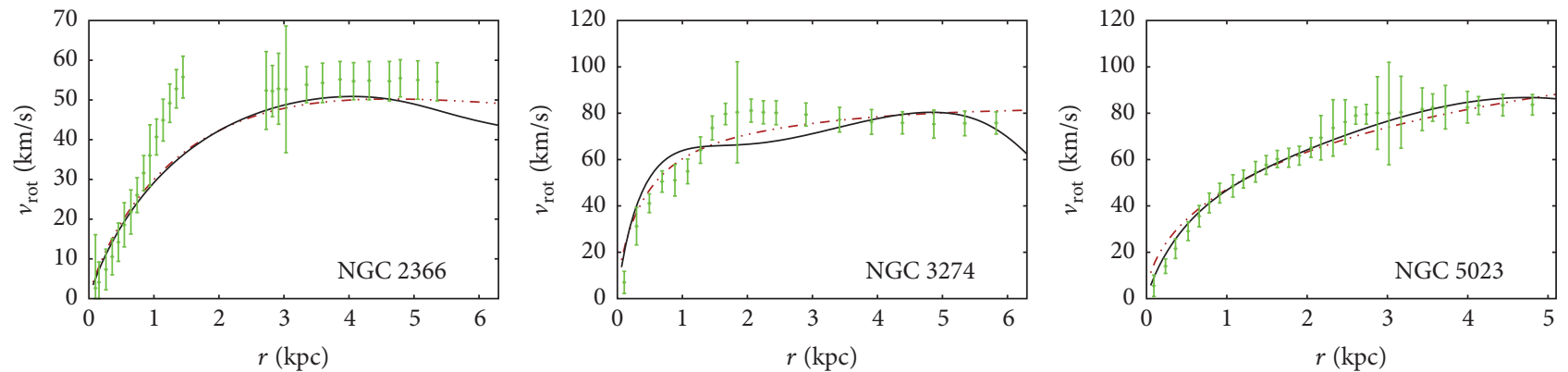

FIGURE 4: Best fit curves for the LSB II. galaxy sample. The solid black lines refer to the baryonic matter + BEC model, while the dashed red lines to the baryonic matter + NFW model. As for HSB galaxies, the BEC model fails to explain the extended flat regions of the rotation curves.

For the LSB I galaxies the BEC DM model gives significantly better fitting velocity curves (all within the $1 \sigma$ confidence level) compared to the NFW model (which in two cases out of the three gives fits falling outside $1 \sigma$ ). For LSB II galaxies the quality of the fits is comparable, but in both models they are beyond the $1 \sigma$ confidence level.

3.3. Dwarf Galaxies. Approximately $85 \%$ of the explored galaxies in the Local Volume [89] are dwarf galaxies. The dwarfs are defined by having an absolute magnitude which is fainter than $M_{B} \sim-16 \mathrm{mag}$. On the other hand they are larger than globular clusters [90].

Although little is known about their formation, it is generally accepted that dwarfs are formed at the centres of subhalos. Dwarf galaxies can be categorised in five groups according to their optical appearance. The five groups being dwarf ellipticals, dwarf irregulars, dwarf spheroidals, blue compact dwarfs, and dwarf spirals. The dwarfs falling in the last group represent the very small ends of spirals [91]. Dwarf spheroidals are old systems and among the most DM dominated galaxies in the Universe.

The central velocity dispersion of most dwarf galaxies is in the range $6 \div 25 \mathrm{~km} / \mathrm{s}$ [92]. In a typical dwarf galaxy, assuming dynamical equilibrium, the mass derived from the observed velocity dispersion is substantially greater than the observed total visible mass. This implies that the mass-to-light ratio is very high compared to other types of galaxies; hence they can greatly contribute to the understanding of DM distribution on small scales. Dwarf galaxies allow for proving or falsifying different alternative gravity theories [93].
We decided to use 7 dwarf galaxies for testing the BEC model. We have selected the sample dwarf galaxies such as to ensure that sufficient high-resolution rotation curve data would be available for our study. We fitted both the BEC + baryonic and the NFW + baryonic models, respectively, with similar baryonic components as for the LSB galaxies. As the length scales of the stellar disks were not available for the selected sample, they were calculated by $\chi^{2}$ minimization, too.

A preliminary check showed that the addition of the BEC dark matter halo to the baryonic model improved (giving lower $\chi_{\min }^{2}$ values) on the fit in all cases. By contrast, the NFW model was unable to improve on the purely baryonic fit in four out of seven cases. We note that since the data does not contain the error margins, the $\chi_{\min }^{2}$ values are relatively high (beyond the $1 \sigma$ confidence level in most cases). The best fit BEC and NFW parameters are shown in Table 4 and the corresponding rotation curves are represented on Figure 5. The inclusion of the BEC DM model gives significantly (in some cases one order of magnitude in the value of $\chi^{2}$ ) better fits compared to the case of NFW model. This is due to the cusp avoidance in the central density profile of the BEC model and the fact that dwarf galaxies do not exhibit extended flat regions in their rotation curves.

\section{Discussions and Final Remarks}

We have performed a $\chi^{2}$-test of the BEC and NFW DM models, with the rotation curves of $6 \mathrm{HSB}, 6 \mathrm{LSB}$, and $7 \mathrm{dwarf}$ 


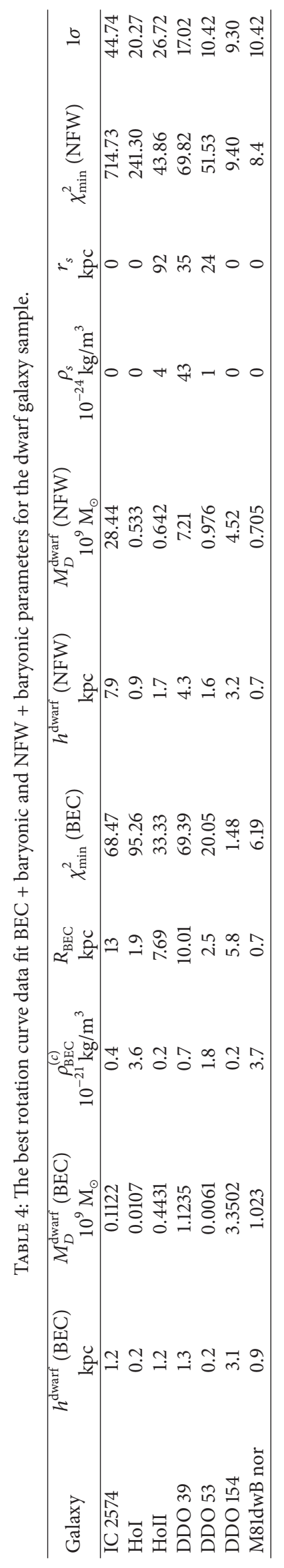



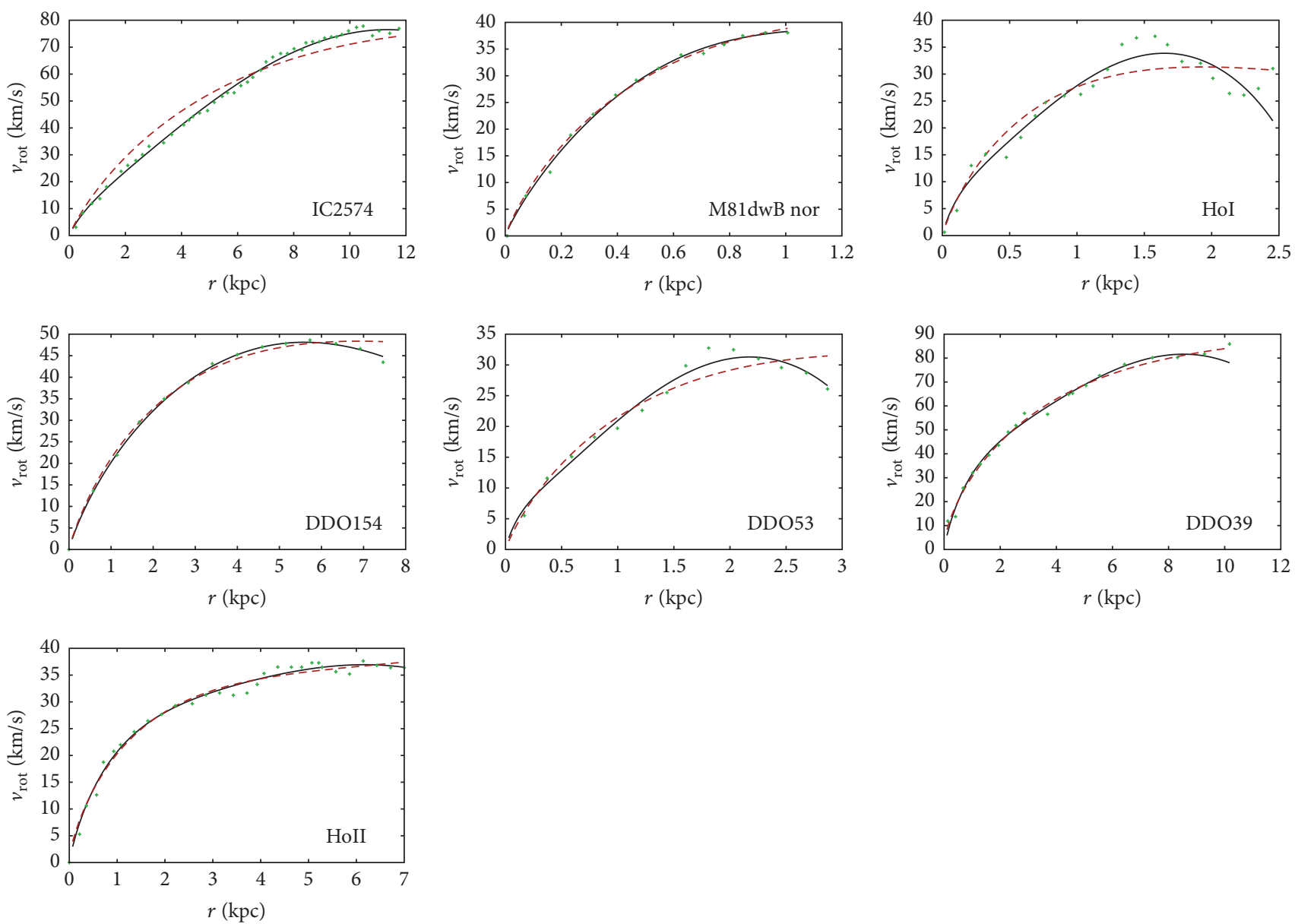

Figure 5: The best fit curves for the dwarf galaxy sample. The BEC + baryonic model (solid black curves) give a better fit in all cases than the NFW + baryonic model (dashed red lines). In both cases the fit was performed with the same baryonic model.

galaxy samples. For improved accuracy we also included realistic baryonic models in every case. For the HSB galaxy sample, both the rotation curve and the surface photometry data were available. Most of the rotation curves were smooth, symmetric, and uniform in quality.

The circular velocity of the investigated galaxies was decomposed into its baryonic and DM contribution: $v_{\text {model }}^{2}(r)=v_{\text {baryonic }}^{2}+v_{\mathrm{DM}}^{2}$. For the BEC model the DM contribution to the rotational velocity can be described as (8). Then the rotation curves are fitted with the parameters of the baryonic and DM halo models (BEC and NFW) using $\chi^{2}$ minimization method.

The analysis of the HSB I galaxies showed a remarkably good agreement for both DM models with observations. The BEC and NFW models show similar fits. However, the rotation curves of the HSB II type galaxies are significantly better described by the NFW model.

It was previously known that for LSB galaxies and without including the baryonic sector, the BEC model gave a better fit than the NFW model [62]. We additionally found that including the baryonic component improves on the fit of [62]. Our detailed analysis showed a significantly better performance of the BEC model for LSB type I galaxies, while comparable fits for LSB type II galaxies were obtained. These latter fits were however outside the $2 \sigma$ confidence level.

The unsatisfactory large distance behaviour of the BEC model for both the HSB and LSB galaxies of type II originates in the sharp cutoff of the BEC DM distribution and clearly indicates that it would be desirable to modify the BEC model on larger scale, also to comply with the behaviour of the universal rotation curves (URCs) at larger radii [94].

From the above analysis of HSB and LSB galaxies it is also obvious that (while on large distances the BEC model suffers from problems due to the sharp cutoff) close to the core it works overall better than the NFW model. This is also supported by our fit of both the BEC + baryonic and NFW + baryonic DM models with rotation curve data of a sample of 7 dwarf galaxies. Since dwarf galaxies are DM dominated, they allow for the best comparison between the various models. The results can be seen in Figure 5. We also note that the NFW DM improved over the pure baryonic fit in four cases out of seven, while including the BEC component improved over the fit with the baryonic component in all cases.

The BEC parameters were determined for all cases. The parameters $\rho_{\mathrm{BEC}}^{(c)}, R_{\mathrm{BEC}}$ are given in Tables 2,3 , and 4 . The averages of the radii $R_{\mathrm{DM}}$ of the BEC halos for the HSB, LSB, 


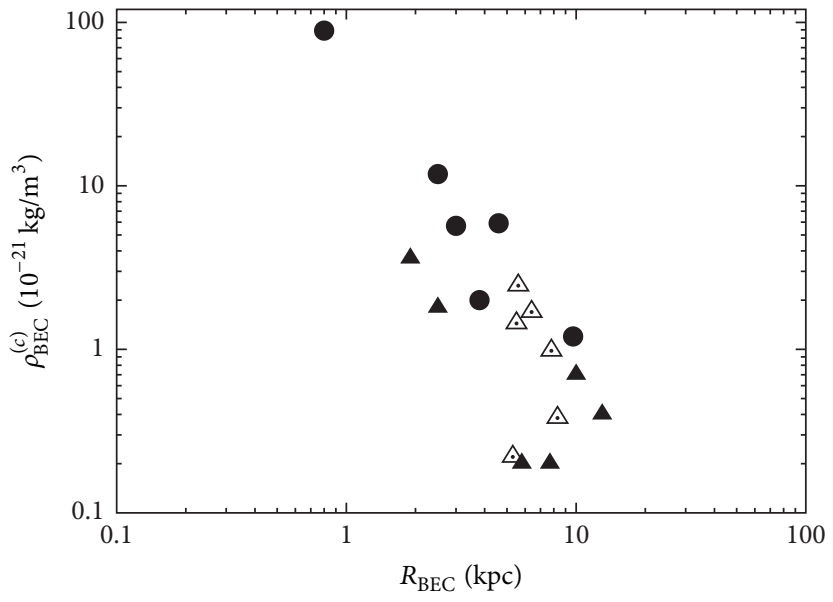

(a)

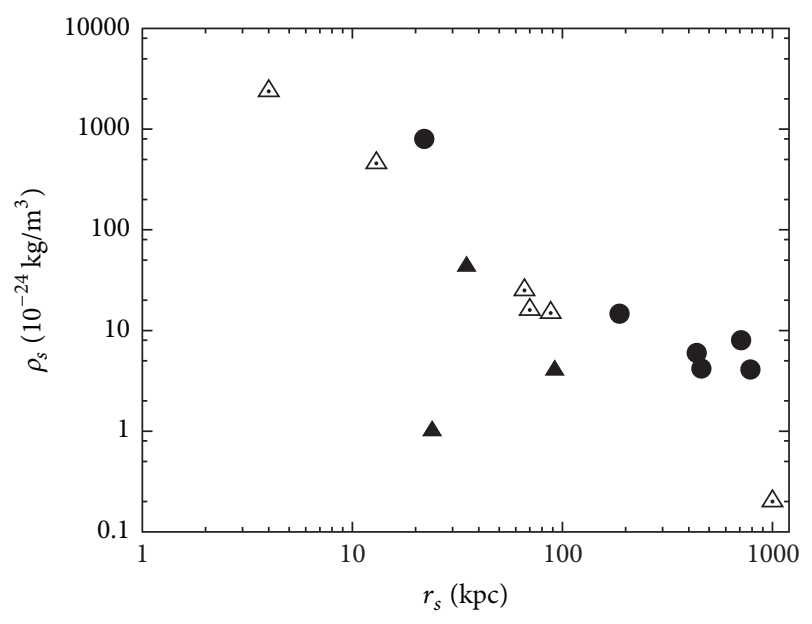

(b)

FIGURE 6: The density parameter $\rho_{\mathrm{BEC}}^{(c)}$ of the BEC model is shown as function of $R_{\mathrm{BEC}}$ in (a), and the density parameter $\rho_{s}$ of the NFW model is shown as function of $r_{s}$ in (b). The HSB, LSB, and dwarf galaxies are represented by filled circles, empty triangles, and filled triangles, respectively.

and dwarf galaxies are $\left\langle R_{\mathrm{BEC}}^{\mathrm{HSB}}\right\rangle \approx 4.06 \mathrm{kpc},\left\langle R_{\mathrm{DM}}^{\mathrm{LSB}}\right\rangle \approx 6.48 \mathrm{kpc}$, and $\left\langle R_{\mathrm{DM}}^{\text {dwarf }}\right\rangle \approx 5.94 \mathrm{kpc}$, respectively. The scatter however is large; there are no universal BEC parameters which globally fit all the galaxies, not even at $3 \sigma$ confidence level. The closer to this goal were the HSB galaxies, where 3 out of 6 had overlapping $3 \sigma$ domains. Nonetheless the given values of $R_{\mathrm{DM}}$ are consistent within the order of magnitude with the halo radii of 59 other galaxies determined from weak lensing [95].

We represent the density parameter $\rho_{\mathrm{BEC}}^{(c)}$ of the BEC model as function of $R_{\mathrm{BEC}}$ in Figure 6(a) and the density parameter $\rho_{s}$ of the NFW model as function of $r_{s}$ in Figure 6(b) (four dwarf galaxies are absent, as the NFW halo does not improve the fit over the pure baryonic case). The fitting enforces a relation between the dark matter parameters: the characteristic density scales with an inverse power with the corresponding characteristic distance.

We verify the Tully-Fisher relation for the investigated galaxy sample and present the results on Figure 7. Apparent $B$ magnitudes and galaxy distances were collected from the NASA/IPAC extragalactic database [96] and were corrected for extinction based on Landolt standard fields to calculate the absolute magnitudes. It is known that the Tully-Fisher relation holds for spiral and lenticular galaxies with the same slope (e.g., [97]). A larger slope and scatter characterize the Tully-Fisher relation for the dwarf galaxies (e.g., $[97,98])$. The investigated sample exactly exhibits these features.

There is a relation among the mass $m$ of the BEC particle, its coherent scattering length $a$, and the radius of the DM halo $R_{\mathrm{DM}}[10]:$

$$
\begin{aligned}
m & =\left(\frac{\pi^{2} \hbar^{2} a}{G R_{\mathrm{BEC}}^{2}}\right)^{1 / 3} \\
& \approx 6.73 \times 10^{-2}[a(\mathrm{fm})]^{1 / 3}\left[R_{\mathrm{BEC}}(\mathrm{kpc})\right]^{-2 / 3} \mathrm{eV} .
\end{aligned}
$$

Axions have been proposed as the Peccei-Quinn solution to the strong CP problem [99] and they are among the best dark

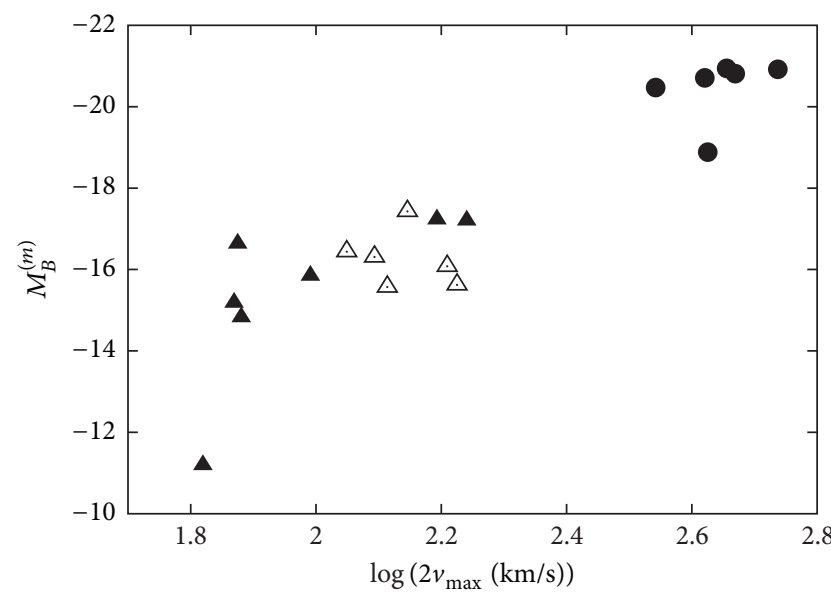

FIGURE 7: The baryonic Tully-Fisher relation of our galaxy sample. Absolute $B$ magnitudes are presented as function of the logarithm of the maximal rotational velocity. The HSB, LSB, and dwarf galaxies are represented by filled circles, empty triangles, and filled triangles, respectively.

matter candidates. Being bosons, they may also form BEC. The Axion Dark Matter Experiment has already established limits on the dark matter axions $[100,101]$.

Assuming the BEC is formed of axions with mass of $10^{-6} \mathrm{eV}$, the scattering lengths for the three types of galaxies emerge as $a_{\mathrm{HSB}} \approx 5.4 \times 10^{-14} \mathrm{fm}, a_{\mathrm{LSB}} \approx 1.37 \times 10^{-13} \mathrm{fm}$, and $a_{\mathrm{dwarf}} \approx 1.15 \times 10^{-13} \mathrm{fm}$. These values are consistent with the results of [95], which are based on a statistical analysis of $61 \mathrm{DM}$ dominated galaxies. The total energy of the BEC halo is negative with these scattering lengths and particle mass, meaning the halo is stable (see Figure 3 of [56]).

\section{Competing Interests}

The authors declare that they have no competing interests. 


\section{Acknowledgments}

In the earlier stages of this work M. Dwornik, Z. Keresztes, and L. Á. Gergely were supported by the European Union and the State of Hungary, cofinanced by the European Social Fund in the framework of TÁMOP 4.2.4. A/2-11-/1-20120001 “National Excellence Program”. L. Á. Gergely was also supported by the Japan Society for the Promotion of Science.

\section{References}

[1] P. A. R. Ade, N. Aghanim, C. Armitage-Caplan et al., "Planck 2013 results. I. Overview of products and scientific results," Astronomy \& Astrophysics, vol. 571, article 1, 2014.

[2] M. Francis, "First Planck results: the Universe is still weird and interesting," Arstechnica, 2013.

[3] J. Ja locha, L. Bratek, M. Kutschera, and P. Skindzier, "Global disc models for galaxies NGC 1365, 6946, 7793 and UGC 6446," Monthly Notices of the Royal Astronomical Society, vol. 406, no. 4, pp. 2805-2816, 2010.

[4] M. Milgrom, "A modification of the Newtonian dynamics as a possible alternative to the hidden mass hypothesis," The Astrophysical Journal, vol. 270, pp. 365-370, 1983.

[5] R. H. Sanders, "Anti-gravity and galaxy rotation curves," Astronomy \& Astrophysics, vol. 136, no. 2, pp. L21-L23, 1984.

[6] J. Moffat and I. Sokolov, "Galaxy dynamics predictions in the nonsymmetric gravitational theory," Physics Letters B, vol. 378, no. 1-4, pp. 59-67, 1996.

[7] P. D. Mannheim, "Are galactic rotation curves really flat?" Astrophysical Journal, vol. 479, no. 2, pp. 659-664, 1997.

[8] M. D. Roberts, "Galactic metrics," General Relativity and Gravitation, vol. 36, no. 11, pp. 2423-2431, 2004.

[9] C. G. Böhmer and T. Harko, "On Einstein clusters as galactic dark matter haloes," Monthly Notices of the Royal Astronomical Society, vol. 379, no. 1, pp. 393-398, 2007.

[10] C. G. Böhmer and T. Harko, "Can dark matter be a BoseEinstein condensate?" Journal of Cosmology and Astroparticle Physics, vol. 2007, no. 6, 2007.

[11] O. Bertolami, C. G. Böhmer, T. Harko, and F. S. Lobo, "Extra force in $\boldsymbol{f}(\boldsymbol{R})$ modified theories of gravity," Physical Review D. Particles, Fields, Gravitation, and Cosmology, vol. 75, no. 10, Article ID 104016, 2007.

[12] C. G. Böhmer, T. Harko, and F. S. N. Lobo, "Dark matter as a geometric effect in f (R) gravity," Astroparticle Physics, vol. 29, no. 6, pp. 386-392, 2008.

[13] M. K. Mak and T. Harko, "Can the galactic rotation curves be explained in brane world models?" Physical Review D, vol. 70, no. 2, Article ID 024010, 2004.

[14] F. Rahaman, M. Kalam, A. DeBenedictis, A. A. Usmani, and R. Saibal, "Galactic rotation curves and brane world models," Monthly Notices of the Royal Astronomical Society, vol. 389, no. 1, pp. 27-33, 2008.

[15] L. Á. Gergely, T. Harko, M. Dwornik, G. Kupi, and Z. Keresztes, "Galactic rotation curves in brane world models," Monthly Notices of the Royal Astronomical Society, vol. 415, no. 4, pp. 3275-3290, 2011.

[16] A. Stabile and S. Capozziello, "Galaxy rotation curves in $f(R, \phi)$ gravity," Physical Review D, vol. 87, no. 6, Article ID 064002, 2013.

[17] E. Conover, "Physicists narrow in on electrical short in Large Hadron Collider," Science, 2015.
[18] N. Arkani-Hamed et al., SUSY Bet: Arkani-Hamed and Panel Discussion, Current Themes in High Energy Physics and Cosmology, Copenhagen, Denmark, 2016.

[19] M. G. Aartsen, K. Abraham, M. Ackermann et al., "Searches for sterile neutrinos with the IceCube detector," Physical Review Letters, vol. 117, no. 7, Article ID 071801, 9 pages, 2016.

[20] D. S. Akerib, H. M. Araujo, X. Bai et al., "First results from the LUX dark matter experiment at the sanford underground research facility," Physical Review Letters, vol. 112, no. 9, Article ID 091303, 2013.

[21] PandaX-II Collaboration, "Dark matter results from First 98.7day data of pandaX-II experiment," Physical Review Letters, vol. 117, Article ID 121303, 2016.

[22] E. Aprile, J. Aalbers, F. Agostini et al., "XENON100 dark matter results from a combination of 477 live days," Physical Review D, vol. 94, no. 12, Article ID 122001, 2016.

[23] D. Choudhury and K. Ghosh, "Bounds on universal extra dimension from LHC run I and II data," Physics Letters B, vol. 763, pp. 155-160, 2016.

[24] C. Alcock, R. A. Allsman, D. R. Alves et al., "The MACHO project: microlensing results from 5.7 years of large magellanic cloud observations," Astrophysical Journal, vol. 542, no. 1, pp. 281-307, 2000

[25] B. P. Abbott, R. Abbott, T. D. Abbott et al., "Observation of gravitational waves from a binary black hole merger," Physical Review Letters, vol. 116, no. 6, Article ID 061102, 2016.

[26] D. O. Caldwell, Current Aspects of Neutrino Physics, Springer, Berlin, Heidelberg, 2001.

[27] H. J. de Vega and N. G. Sanchez, "Warm dark matter in the galaxies: theoretical and observational progresses. Highlights and conclusions of the chalonge meudon workshop 2011," https://arxiv.org/abs/1109.3187.

[28] H. Wei, Z.-C. Chen, and J. Liu, "Cosmological constraints on variable warm dark matter," Physics Letters B, vol. 720, no. 4-5, pp. 271-276, 2013.

[29] P. L. Biermann and A. Kusenko, "Relic keV sterile neutrinos and reionization," Physical Review Letters, vol. 96, no. 9, Article ID 091301, 2006.

[30] M. G. Aartsen, M. Ackermann, J. Adams et al., "Searches for sterile neutrinos with the icecube detector," Physical Review Letters, vol. 117, no. 7, Article ID 071801, 2016.

[31] T. Padmanabhan, "Cosmological constant-the weight of the vacuum," Physics Reports. A Review Section of Physics Letters, vol. 380, no. 5-6, pp. 235-320, 2003.

[32] P. J. Peebles and B. Ratra, "The cosmological constant and dark energy," Reviews of Modern Physics, vol. 75, no. 2, pp. 559-606, 2003.

[33] A. Fowlie, K. Kowalska, L. Roszkowski, E. M. Sessolo, and Y.L. S. Tsai, "Dark matter and collider signatures of the MSSM," Physical Review D, vol. 88, no. 5, Article ID 055012, 2013.

[34] G. Aad, T. Abajyan, B. Abbott et al., "Search for invisible decays of a higgs boson produced in association with a $Z$ boson in ATLAS," Physical Review Letters, vol. 112, no. 20, Article ID 201802, 19 pages, 2014.

[35] P. H. Frampton, "Angular momentum of dark matter black holes," https://arxiv.org/abs/1608.05009.

[36] M. Sasaki, T. Suyama, T. Tanaka, and S. Yokoyama, "Primordial black hole scenario for the gravitational-wave event GW150914," Physical Review Letters, vol. 117, no. 6, Article ID 061101, 2016.

[37] P. Palunas and T. B. Williams, "Maximum disk mass models for spiral galaxies," Astronomical Journal, vol. 120, no. 6, pp. 28842903, 2000. 
[38] W. J. G. de Blok and A. Bosma, "High-resolution rotation curves of low surface brightness galaxies," Astronomy and Astrophysics, vol. 385, no. 3, pp. 816-846, 2002.

[39] V. Springel, S. D. M. White, A. Jenkins et al., "Simulations of the formation, evolution and clustering of galaxies and quasars," Nature, vol. 435, no. 7042, pp. 629-636, 2005.

[40] J. F. Navarro, C. S. Frenk, and S. D. M. White, "The structure of cold dark matter halos," Astrophysical Journal, vol. 462, no. 2, pp. 563-575, 1996.

[41] O. Valenzuela, G. Rhee, A. Klypin et al., "Is there evidence for flat cores in the halos of dwarf galaxies? The case of NGC 3109 and NGC 6822," Astrophysical Journal, vol. 657, no. 2 I, pp. 773789, 2007.

[42] J. R. Jardel, K. Gebhardt, M. H. Fabricius, N. Drory, and M. J. Williams, "Measuring dark matter profiles non-parametrically in dwarf spheroidals: an application to Draco," Astrophysical Journal, vol. 763, no. 2, article 91, 2013.

[43] A. Burkert, "The structure of dark matter halos. Observation versus theory," in Dark Matter in Astro- and Particle Physics: (DARK '96): Heidelberg, Germany, 16-20 September 1996, H. V. Klapdor-Kleingrothaus and Y. Ramachers, Eds., p. 35, World Scientific, Singapore, 1997.

[44] R. Teyssier, A. Pontzen, Y. Dubois, and J. I. Read, "Cusp-core transformations in dwarf galaxies: observational predictions," Monthly Notices of the Royal Astronomical Society, vol. 429, no. 4, pp. 3068-3078, 2013.

[45] S. Inoue and T. R. Saitoh, "Cores and revived cusps of dark matter haloes in disc galaxy formation through clump clusters," Monthly Notices of the Royal Astronomical Society, vol. 418, no. 4, pp. 2527-2531, 2011.

[46] A. Klypin and F. Prada, "Testing gravity with motion of satellites around galaxies: Newtonian gravity against modified Newtonian dynamics," Astrophysical Journal, vol. 690, no. 2, pp. 1488-1496, 2009.

[47] L. Á. Gergely and S. Tsujikawa, "Effective field theory of modified gravity with two scalar fields: dark energy and dark matter," Physical Review D, vol. 89, no. 6, Article ID 064059, 2014.

[48] I. Rodríguez-Montoya, J. Magaña, T. Matos, and A. PérezLorenzana, "Ultra light bosonic dark matter and cosmic microwave background," Astrophysical Journal, vol. 721, no. 2, pp. 1509-1514, 2010.

[49] S.-J. Sin, "Late-time phase transition and the galactic halo as a Bose liquid," Physical Review D, vol. 50, no. 6, article 3650, 1994.

[50] P. Sikivie, "Caustic rings of dark matter," Physics Letters B, vol. 432, no. 1-2, pp. 139-144, 1998.

[51] P. Sikivie, "Caustic ring singularity," Physical Review DParticles, Fields, Gravitation and Cosmology, vol. 60, no. 6, pp. 1-16, 1999.

[52] E. P. Gross, "Structure of a quantized vortex in boson systems," Nuovo Cimento, vol. 20, no. 3, pp. 454-477, 1961.

[53] E. P. Gross, "Hydrodynamics of a superfluid condensate," Journal of Mathematical Physics, vol. 4, no. 2, p. 195, 1963.

[54] L. P. Pitaevskii, "Vortex lines in an imperfect bose gas," Zhurnal Éksperimental'noй i Teoreticheskoй Fiziki, vol. 40, no. 2, p. 646, 1961.

[55] T. Harko, "Cosmological dynamics of dark matter Bose-Einstein condensation," Physical Review D, vol. 83, no. 12, Article ID 123515, 2011.

[56] J. C. C. De Souza and M. O. C. Pires, "Discussion on the energy content of the galactic dark matter Bose-Einstein condensate halo in the Thomas-Fermi approximation," Journal of Cosmology and Astroparticle Physics, vol. 2014, no. 3, article no. 010, 2014.

[57] T. Harko, "Gravitational collapse of Bose-Einstein condensate dark matter halos," Physical Review D-Particles, Fields, Gravitation and Cosmology, vol. 89, no. 8, Article ID 084040, 2014.

[58] H. Velten and E. Wamba, "Power spectrum for the BoseEinstein condensate dark matter," Physics Letters, Section B: Nuclear, Elementary Particle and High-Energy Physics, vol. 709, no. 1-2, pp. 1-5, 2012.

[59] J. W. Lee, S. Lim, and D. Choi, "BEC dark matter can explain collisions of galaxy clusters," https://arxiv.org/abs/0805.3827.

[60] D. Boyanovsky, H. J. De Vega, and N. G. Sanchez, "Constraints on dark matter particles from theory, galaxy observations, and N-body simulations," Physical Review D, vol. 77, no. 4, Article ID 043518, 2008.

[61] A. X. González-Morales, A. Diez-Tejedor, L. A. Ureña-López, and O. Valenzuela, "Hints on halo evolution in scalar field dark matter models with galaxy observations," Physical Review D, vol. 87, no. 2, 2013.

[62] V. H. Robles and T. Matos, "Flat central density profile and constant dark matter surface density in galaxies from scalar field dark matter," Monthly Notices of the Royal Astronomical Society, vol. 422, no. 1, pp. 282-289, 2012.

[63] M. Dwornik, Z. Keresztes, and L. Á. Gergely, "Rotation curves in bose-einstein condensate dark matter halos," in Recent Development in Dark Matter Research, N. Kinjo and A. Nakajima, Eds., pp. 195-219, Nova Science, 2014.

[64] L. P. Pitaevskii and S. Stringari, Bose-Einstein Condensation, Oxford University Press, New York, NY, USA, 2003.

[65] S. Grossmann and M. Holthaus, "On Bose-Einstein condensation in harmonic traps," Physics Letters A, vol. 208, no. 3, pp. 188-192, 1995.

[66] W. Ketterle and N. J. van Druten, “Two-step condensation of the ideal bose gas in highly anisotropic traps," Physical Review A, vol. 54, p. 656, 1996.

[67] K. Kirsten and D. J. Toms, "Bose-Einstein condensation of atomic gases in a general harmonic-oscillator confining potential trap," Physical Review A-Atomic, Molecular, and Optical Physics, vol. 54, no. 5, article 4188, 1996.

[68] H. Haugerud, T. Haugset, and F. Ravndal, "A more accurate analysis of Bose-Einstein condensation in harmonic traps," Physics Letters, Section A: General, Atomic and Solid State Physics, vol. 225, no. 1-3, pp. 18-22, 1997.

[69] S. Giorgini, L. P. Pitaevskii, and S. Stringari, "Theory of ultracold atomic Fermi gases," Reviews of Modern Physics, vol. 80, no. 4, 2008.

[70] K. Glaum, A. Pelster, H. Kleinert, and T. Pfau, "Critical temperature of weakly interacting dipolar condensates," Physical Review Letters, vol. 98, no. 8, Article ID 080407, 2007.

[71] M. Schütte and A. Pelster, "Critical temperature of a boseeinstein condensate with $1 / \mathrm{r}$ interactions," in Proceedings of the 9th International Conference on Path Integrals: New Trends and Perspectives (PI '07), W. Janke and A. Pelster, Eds., pp. 417-420, World Scientific, Dresden, Germany, September 2007.

[72] F. Dalfovo, S. Giorgini, L. P. Pitaevskii, and S. Stringari, “Theory of Bose-Einstein condensation in trapped gases," Reviews of Modern Physics, vol. 71, no. 3, pp. 463-512, 1999.

[73] M. H. Anderson, J. R. Ensher, M. R. Matthews, C. E. Wieman, and E. A. Cornell, "Observation of Bose-Einstein condensation in a dilute atomic vapor," Science, vol. 269, no. 5221, pp. 198-201, 1995. 
[74] D. J. Han, R. H. Wynar, P. Courteille, and D. J. Heinzen, “BoseEinstein condensation of large numbers of atoms in a magnetic time-averaged orbiting potential trap," Physical Review AAtomic, Molecular, and Optical Physics, vol. 57, no. 6, pp. R4114R4117, 1998.

[75] U. Ernst, A. Marte, F. Schreck, J. Schuster, and G. Rempe, "Bose-Einstein condensation in a pure Ioffe-Pritchard field configuration," Europhysics Letters, vol. 41, no. 1, pp. 1-6, 1998.

[76] K. B. Davis, M.-O. Mewes, M. R. Andrews et al., "Bose-Einstein condensation in a gas of sodium atoms," Physical Review Letters, vol. 75 , no. 22, article 3969, 1995.

[77] L. V. Hau, B. D. Busch, C. Liu, Z. Dutton, M. M. Burns, and J. A. Golovchenko, "Near-resonant spatial images of confined Bose-Einstein condensates in a 4-Dee magnetic bottle," Physical Review A, vol. 58, no. 1, pp. R54-R57, 1998.

[78] C. C. Bradley, C. A. Sackett, J. J. Tollett, and R. G. Hulet, "Evidence of Bose-Einstein condensation in an atomic gas with attractive interactions," Physical Review Letters, vol. 75, no. 9, pp. 1687-1690, 1995.

[79] E. Madelung, "Quantum theory in hydrodynamic form," Zeitschrift für Physik, vol. 40, pp. 322-326, 1926.

[80] S. Sonego, "Interpretation of the hydrodynamical formalism of quantum mechanics," Foundations of Physics. An International Journal Devoted to the Conceptual Bases and Fundamental Theories of Modern Physics, Biophysics, and Cosmology, vol. 21, no. 10, pp. 1135-1181, 1991.

[81] X. Z. Wang, "Cold Bose stars: self-gravitating Bose-Einstein condensates," Physical Review D, vol. 64, no. 12, Article ID 124009, 2001.

[82] E. H. Lieb, R. Seiringer, and J. Yngvason, "A rigorous derivation of the Gross-Pitaevskii energy functional," Physical Review A, vol. 61, no. 4, Article ID 043602, 2000.

[83] J. L. Sérsic, Atlas de Galaxias Australes, Observatorio Astronomico, Cordoba, Argentina, 1968.

[84] K. C. Freeman, "On the disks of spiral and so galaxies," The Astrophysical Journal, vol. 160, p. 811, 1970.

[85] C. Impey and G. Bothun, "Low surface brightness galaxies," Annual Review of Astronomy and Astrophysics, vol. 35, no. 1, pp. 267-307, 1997.

[86] S. S. Mcgaugh, "Oxygen abundances in low surface brightness disk galaxies," Astrophysical Journal, vol. 426, no. 1, pp. 135-149, 1994.

[87] K. O’Neil, G. D. Bothun, J. Schombert, M. E. Cornell, and C. D. Impey, "A wide field ccd survey for low surface brightness galaxies. II. Color distributions, stellar populations, and missing baryons," Astronomical Journal, vol. 114, no. 6, p. 2448, 1997.

[88] M. Beijersbergen, W. J. G. De Blok, and J. M. Van Der Hulst, "Surface photometry of bulge dominated low surface brightness galaxies," Astronomy and Astrophysics, vol. 351, no. 3, pp. 903919, 1999.

[89] I. D. Karachentsev, V. E. Karachentseva, W. K. Huchtmeier, and D. I. Makarov, "A catalog of neighboring galaxies," Astronomical Journal, vol. 127, no. 4, pp. 2031-2068, 2004.

[90] G. A. Tammann, "Dwarf galaxies in the past," in Dwarf Galaxies, ESO Conference and Workshop Proc No. 49, p. 3, 1994.

[91] L. D. Matthews and J. S. Gallagher III, "B and V CCD photometry of southern, extreme late-type spiral galaxies," Astronomical Journal, vol. 114, no. 5, pp. 1899-1919, 1997.

[92] M. Mateo, "Dwarf galaxies of the local group," Annual Review of Astronomy and Astrophysics, vol. 36, no. 1, pp. 435-506, 1998.
[93] S. Capozziello, V. F. Cardone, and A. Troisi, "Low surface brightness galaxy rotation curves in the low energy limit of $R^{n}$ gravity: no need for dark matter?" Monthly Notices of the Royal Astronomical Society, vol. 375, no. 4, pp. 1423-1440, 2007.

[94] M. Persic, P. Salucci, and F. Stel, “The universal rotation curve of spiral galaxies-I. The dark matter connection," Monthly Notices of the Royal Astronomical Society, vol. 281, no. 1, pp. 27-47, 1996.

[95] M. O. C. Pires and J. C. C. De Souza, "Galactic cold dark matter as a Bose-Einstein condensate of WISPs," Journal of Cosmology and Astroparticle Physics, vol. 2012, no. 11, article 024, 2012.

[96] G. Helou, B. F. Madore, M. Schmitz, M. D. Bicay, X. Wu, and J. Bennett, "The NASA/IPAC extragalactic database," in Databases \& On-Line Data in Astronomy, vol. 171 of Astrophysics and Space Science Library, pp. 89-106, Springer Netherlands, 1991.

[97] S. S. Mcgaugh, J. M. Schombert, G. D. Bothun, and W. J. G. De Blok, "The baryonic tully-fisher relation," Astrophysical Journal, vol. 533, no. 2, pp. L99-L102, 2000.

[98] S. S. McGaugh and J. Olf, "Local group dwarf spheroidals: correlated deviations from the Baryonic Tully-Fisher Relation," Astrophysical Journal, vol. 722, no. 1, pp. 248-261, 2010.

[99] R. D. Peccei and H. R. Quinn, "CP conservation in the presence of pseudoparticles," Physical Review Letters, vol. 38, no. 25, pp. 1440-1443, 1977.

[100] S. J. Asztalos, G. Caosi, C. Hagmann et al., “The Axion Dark Matter eXperiment," in Proceedings of the 31st International Symposium on Physics in Collision, Vancouver, Canada, August 2011.

[101] L. J. Rosenberg, "Dark-matter QCD-axion searches," Proceedings of the National Academy of Sciences of the United States of America, vol. 112, no. 40, pp. 12278-12281, 2015. 

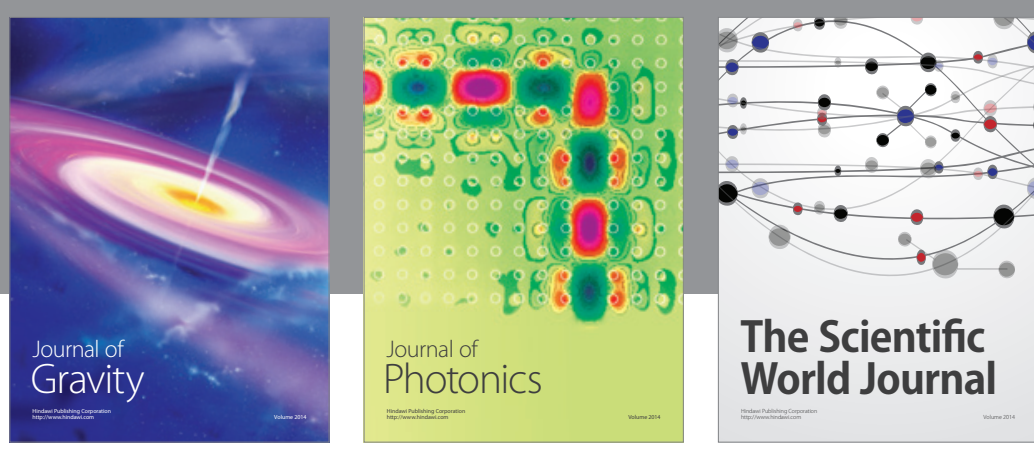

The Scientific World Journal
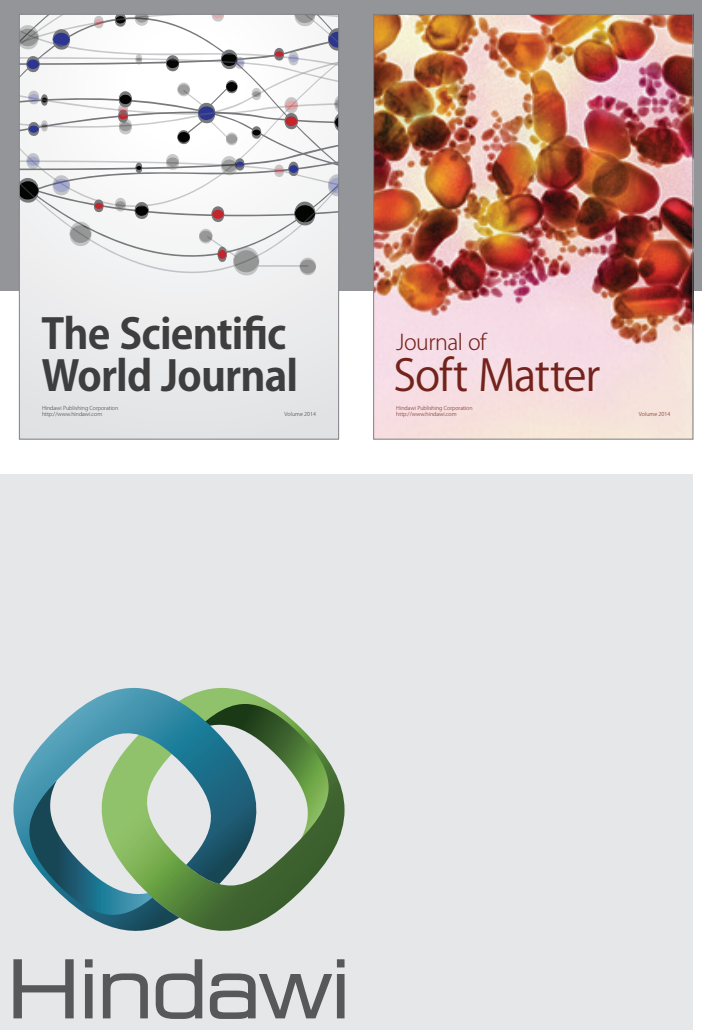

Submit your manuscripts at

https://www.hindawi.com
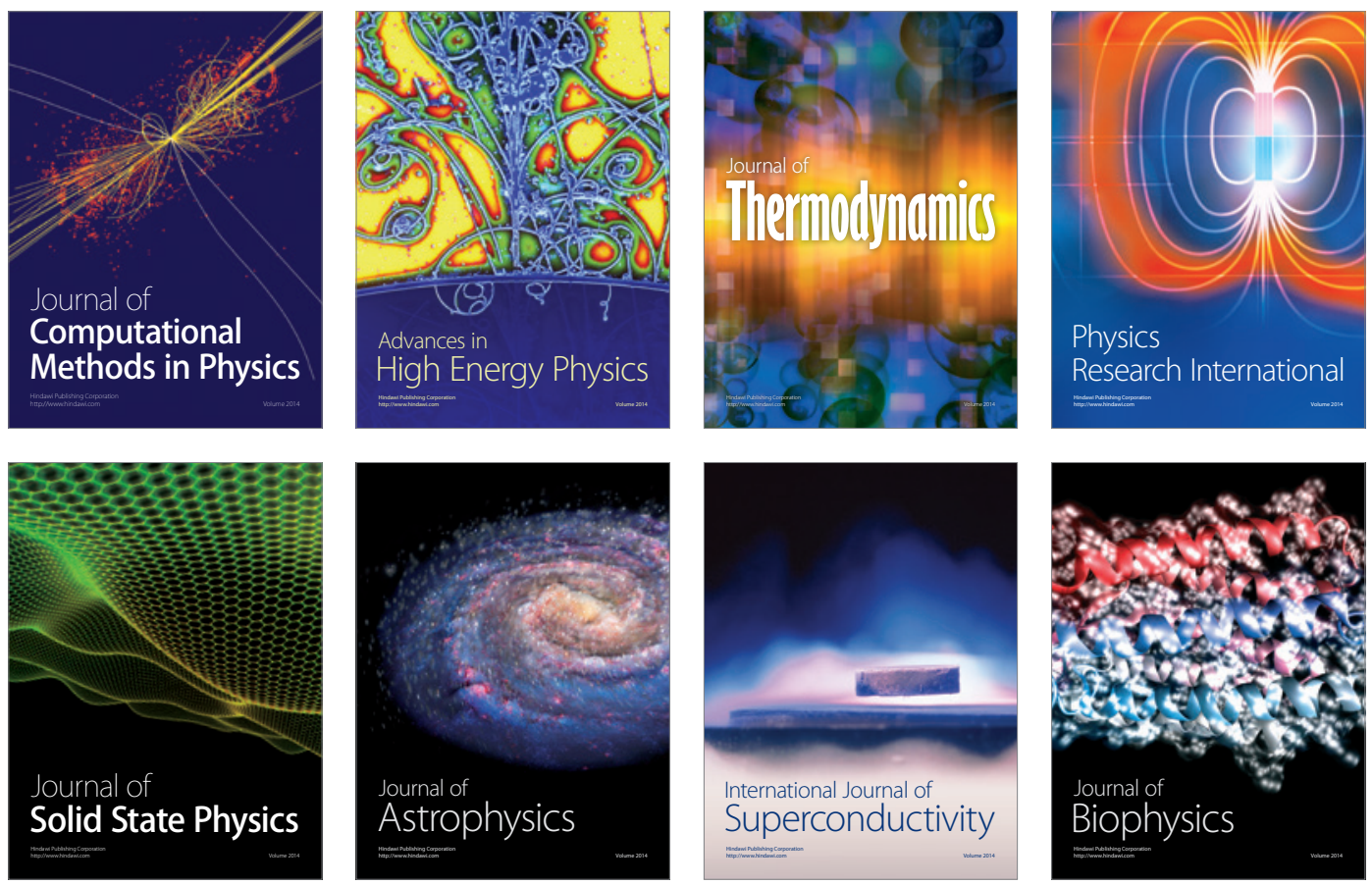
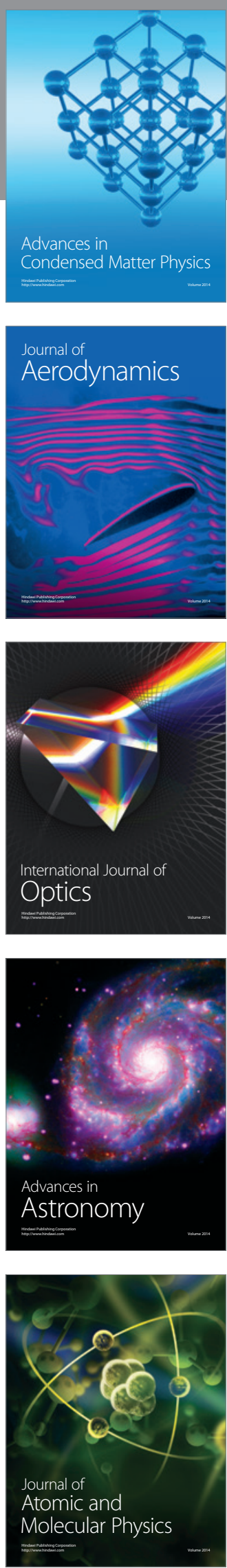